\title{
Unifying multi-level modeling: A position paper
}

\author{
Manfred A. Jeusfeld \\ School of Informatics \\ University of Skövde \\ Skövde, Sweden \\ Email: manfred.jeusfeld@his.se \\ ORCID 0000-0002-9421-8566
}

\author{
Ulrich Frank \\ Institute for Computer Science \& Business Informatics \\ University of Duisburg-Essen \\ Essen, Germany \\ Email: ulrich.frank@uni-due.de \\ ORCID 0000-0002-8057-1836
}

\begin{abstract}
Multi-level modeling (MLM) as part of objectoriented modeling aims at fully utilizing the expressive power of multiple abstraction levels. While these levels where initially used to define domain-specific modeling languages, i.e. for linguistic purposes, the MLM community has long argued that there is much more to gain by tapping into ontological abstraction levels. While MLM is a rather specialized research field, there are now quite a number of different proposals. There is thus an opportunity to develop a uniform core of MLM that then possibly can become part of a standard and be taken up by the larger modeling community.
\end{abstract}

Keywords-multi-level modeling, conceptual modeling, research agenda

\section{INTRODUCTION}

Multi-level modeling (MLM) was introduced at the beginning of the new millennium [1], with roots that go back to the nineties of last century (cf. [2]-[5]). In the meantime a variety of approaches that comprise specific languages and tools, has evolved. Most of these approaches are based on object-oriented constructs cf., e.g., [6]-[10]. The second category of multilevel languages is set- or logic-based, with some that have a specific focus on data or knowledge bases, cf. [5], [11], [12].

While all these approaches seem to be driven by the same motivation, that is, to promote reuse and maintainability of models and systems through additional abstraction, they are characterized by a remarkable diversity with respect to terminology and implementation languages. Even though some of these differences turn out to be marginal on closer inspection, we regard the diversity of the field as problematic. On the one hand, the lack of a unified, not to speak of a standardized core, is a clear obstacle to adoption and dissemination of MLM. On the other hand, this kind of diversity [13] also makes fruitful competition in research difficult.

In this position paper, we present the the draft of a roadmap that outlines how we as a community might overcome the current, as we perceive it, unsatisfactory state of the field. It is not meant as a recommendation, but as a proposal to inspire a discussion on the future of the field. The paper starts with theses that reflect our assessment of our community. Based on this analysis, we present cornerstones of a common roadmap together with ideas of how to implement it. We are aware of the fact that a roadmap or some kind of memorandum calls for the participation of more members of the MLM community to serve as a representative and accepted orientation. Unfortunately, we did not have enough time to organize such an initiative prior to the workshop. Therefore, we hope that the position paper is perceived as a first input to a discussion during the workshop that hopefully results in a consolidated roadmap that serves us as a community as an orientation.

\section{Some Theses on the Current State}

We have been devoted members of the MLM community for some time. We regard the fundamental ideas that carry the community as extremely important for advancing the fields of conceptual modeling in particular and of software engineering in general. We enjoyed the discussions we experienced during events like the MULTI workshops or a Dagstuhl seminar. Nevertheless, we are concerned. The following theses serve to represent our assessment of the current state. They reflect our opinion, which may not be shared in part by others. Therefore, they are hopefully suited to foster a discussion during and beyond the workshop.

\section{What we like:}

Common conviction. The MLM community is united by the strong, shared conviction that the additional abstraction enabled by multi-level modeling is suited to overcome serious obstacles of the dominating modeling paradigm and is, thus, suited to promote the design of systems that benefit from a higher level of reuse, integration and adaptability.

No fad. The field of IT is characterized by waves of "innovations" that attract a lot of awareness (both in practice and academia), but often for a short time only, because they get demystified and/or people loose interest. While MLM never made it to the list of "hot" topics, it is still on the agenda of ambitious researchers that are excited about its potential.

Persistence. Often, research agendas are driven by opportunities like publishing or external funding. Even though respective incentives have been weak so far, members of the MLM community stay committed

Openness. Even though it is united in its criticism of the dominating paradigm, the MLM community does not at all act like members of a sect who close themselves off from other communities. Instead, the community consists of researchers who are respected members of other communities as well.

No hostile environment. MLM is neither perceived as threat 
within other communities, nor is its claim to overcome limitations of the current paradigm seen as offensive.

Never-ending source of research questions: We are only at the beginning of understanding the potential of multi-level modeling and with every new feature we develop, with every case we apply it to, we discover new, inspiring research questions that often go beyond the specific scope of multilevel modeling.

What makes us think:

Widely unknown. Even though a considerable amount of work on MLM has been published in outlets outside our community, it seems that not too many researchers in computer science or business informatics are aware of MLM or are interested in developing a better understanding. It is to be feared that the situation is worse in practice. The lack of awareness is an obstacle to attracting new members and to the acquistion of external funds.

No curriculum. There is no common curriculum that represents the basics of MLM. We even have doubts that MLM is being taught to a considerable extent at all. This creates a serious threat to the future development of the field.

Unsatisfactory exchange. Even though the community is small, the exchange among its members is hampered by heterogeneous terminologies, concepts, and perspectives. While diversity has its merits and is needed to a certain degree, it leads to misunderstanding, creates an obstacle to effective communication and, thus, is an obstacle to advancing the field.

Small community size. The number of researchers in the MLM community is rather small and not growing. This leads to problems in the evaluation and propagation of research results.

Slow progress. Most researchers in the field of MLM focus on their own languages and tools. We assume that many suffer from the lack of resources required to develop their tools. As far as our tools are concerned, we had to learn that under these restrictions (visible) progress takes very long and hinder the creation of success stories.

\section{OUTLINE OF A ROADMAP}

Our brief characterization of the current state, which may not be agreed upon by all members of our community, shows problems and challenges that call for action unless we do not want to jeopardize the future of the field. The following roadmap represents activities that we regard as important to strengthen MLM. The roadmap, which it outlined in fig. 1, is not meant as a process that puts the proposed activities in a strict order. However, some activities are needed to set the stage for others.

\section{A. Emphasis on Commonalities}

Despite the often subtle differences between specific approaches to MLM, there are essential commonalities. We believe that it is important to emphasize and further develop these commonalities. At first, there is the common conviction that MLM offers clear and relevant advantages over the current paradigm. However, except for pointing at the reduction of

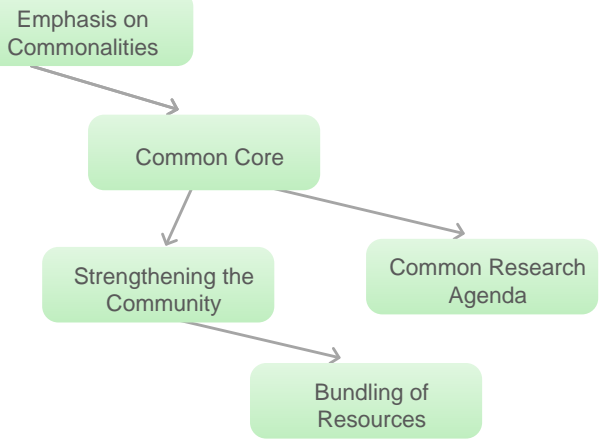

Fig. 1. Roadmap: illustration of cornerstones

accidental complexity, there is only little discussion about further specific benefits.

Proposal: To strengthen the sense of a common foundation, more work and accompanying discussions on specific prospects and challenges of MLM are required.

Second, there is already a wide consensus on basic concepts of MLM. Based on various discussions, in particular during the MULTI workshops or the MLM Dagstuhl seminar [14], we assume there is a wide consensus about the essential characteristics of MLM.

1) MLM allows for an arbitrary number of abstraction levels that are not created through generalization alone. Note that we refrain from using the term "classification levels" for reasons explained below.

2) Every class in a multi-level model is an object at the same time. That is, may have state. Differences: Some approaches also provide for objects to execute operations.

3) The instantiation of properties of classes on level m can be deferred to classes on levels below m-1. Differences: While deferred instantiation of attributes is a common characteristic, deferred instantiation of operations and associations is not supported by all approaches.

Proposal: These commonalities should be discussed and, if needed, refined in order to make them a trusted common reference. In addition, those aspects that represent differences, should also be clearly identified and described with respect to their essential features. It might also be an issue to consider the extension of multi-level language architectures to programming languages, which would recommend defining essential characteristics of multi-level programming languages.

It is a common characteristic of a new paradigm that the terminology used within a previous paradigm is not appropriate any more. In the case of MLM, this creates a particular problem. Most members of the MLM community are active members of other communities that are committed to the previous, and still existing (!), paradigm. Therefore, it is not about replacing an outdated terminology, but about enabling a consistent co-existence. That suggests for aiming at a monotonic extension of the dominant terminology.

To illustrate how a unified terminology of this kind could 
be conceptualized, we look at a few key concepts of MLM in comparison to corresponding concepts of the traditional paradigm.

Class, Object: In MLM every class is an object at the same time. Since this is not necessarily the case with traditional object-oriented languages, it makes sense to use the term "clabject" introduced by Atkinson and Kühne [1] instead. However, it would not exactly be compatible with the traditional paradigm, since classes on level 1 were regarded as objects in foundational work on object-oriented languages, e.g. in Smalltalk, too.

Instantiation: The semantics of instantiation is different from that in the traditional paradigm. Therefore, using the term "instantiation" would be misleading. Following a proposal made by Neumayr et al. [11], one could use "concretization" instead, while still keeping "instantiation" for cases where it applies.

Classification: For the same reasons as with instantiation, "classification" would be misleading in MLM. Finding an appropriate, that is, self-explanatory term, is demanding. Frank and Töpel suggest "intrinsic classification" [15, p. 2].

Level: The concept of a level is essential. Some approaches use specific concepts to explicitly define the level of a class (see fig. 2) as a number, such as "potency" [1], which describes how often a class and its concretions can be concretized or "level" [7]. Other approaches use designators to characterize levels [11] or do without explicit levels [16].

Proposal: The community should aim at a common terminology by starting with existing proposals such as, e.g., [17], [18], and extend the list step by step. While a unified terminology would be of great value, a certain degree of variety may not only be acceptable, but useful. The terms "potency", for example, and "level" allow for mutual, equivalent replacement. However, they are suited to express different perspectives on the same kind of abstraction.

The relevance of a unified notation is ambivalent. On the one hand, the UML serves as a convincing example, the concrete syntax of a language shapes its perception. On the other hand, it can be seen as a matter of taste and subjective preference. For that reason, it may be useful to offer alternative notations to choose from. In that case, it is important to clearly relate different notations to one another. Fig. 2 illustrates how this can be achieved with three selected MLM notations. A further important reason for relaxing the quest for a unified concrete syntax is the fact that MLM provides an excellent foundation for developing DSML, which will usually features a specific notation anyway.

Proposal: With respect to the benefits of a unified notation, the community should try to agree upon a basic notation. The notation could come in different flavours (e.g. one offering levels, while another one may use potencies). At best, tools would give users the choice between these different flavours.

A strong scientific community needs a unified curriculum that defines how to teach its foundations.

Proposal: The community publishes a call for curriculum proposals. A discussion during MULTI 2022 serves to evaluate

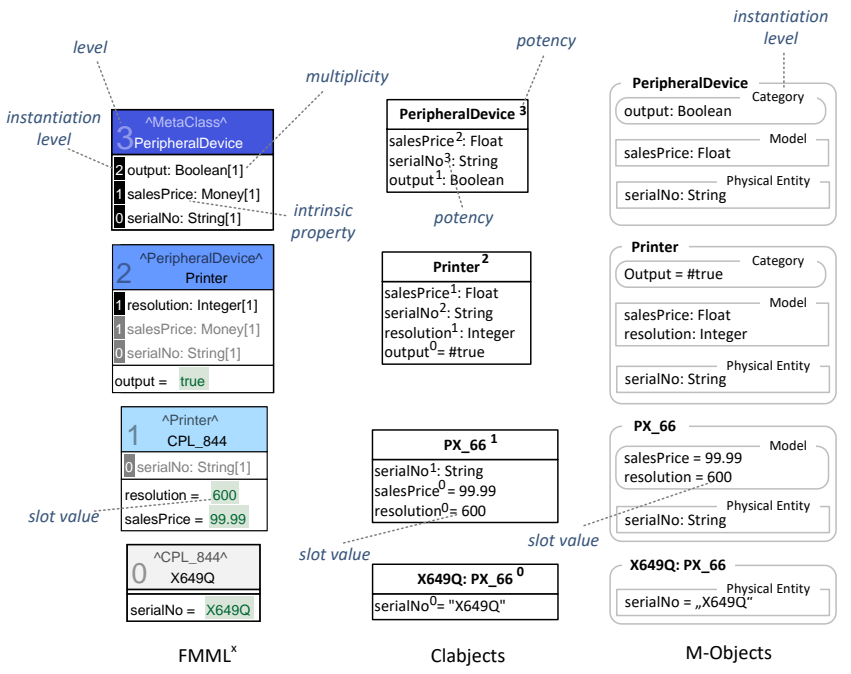

Fig. 2. Corresponding multi-level concepts presented with different notations

submitted proposals and could serve as a starting point for integrating the proposals into a consolidated reference curriculum.

\section{B. Common Core}

Unification and standardization are of pivotal relevance with respect to the adoption and dissemination of new technologies. They promote economies of scale, foster the protection of investments, and, especially with respect to language, create additional value by contributing to a larger number of users. However, especially standardization is likely to bring with it a serious disadvantage. It tends to "freeze" a certain state of the art, which can be a serious obstacle to progress. The dominant object-oriented language paradigm provides a vivid and, we think, cautionary example of this. From an economic point of view the possible drawbacks of standardization might be regarded as acceptable trade-off. This is clearly different in research, where it would contradict the idea of continuously challenging the state of the art. Nevertheless, scientific competition also requires a common ground that facilitates efficient communication and collaboration.

Proposal: The MLM community should strive at a common core of MLM languages, that is, a minimal meta model that serves as a common foundation for more specific language dialects and as a medium to integrate tools.

The identification of commonalities shall serve as a good starting point for developing a common core. We believe that such a common core is suited to make an impact on the larger (object-oriented) modeling community and also to promote collaboration among researchers in the MLM field.

A platform for consensus: The MULTI workshop is a wellestablished platform to discuss issues around MLM. It may thus well kickstart the effort to build a common MLM Core. If a sufficient number of researchers from the MLM community agree to form a task force, then this task force should be assigned to make a first proposal. We suggest the following principles of the task force: 
1) The members should be active in the MLM or modeling community, ideally having designed/implemented/applied related modeling languages.

2) Membership should be inclusive and open.

3) The task force should aim at a minimal compromise for MLM Core, not at a comprehensive framework attempting to cover all constructs discussed to far.

The work of the task force could be organized similar to the MULTI challenges. However, there would only be a single deliverable, MLM Core, being produced by all members of the task force. Note that the MULTI challenges were designed to test the expressiveness of existing MLM proposals. MLM Core would not be the most expressive MLM framework. It serves as the minimal baseline, to which more elaborate proposals can refer to enhance interoperability and re-usability.

\section{Milestones}

The following milestones should be targeted to achieve this goal.

1) Define the constructs of MLM Core and their precise semantics. In particular, clarify whether there is a need for two separate constructs for instantiation. The semantics should be capable to distinguish sound from unsound models.

2) Define the translation of existing MLM models representing in the competing approaches to MLM Core. Note that that not all constructs in the existing MLM models may be supported by MLM Core.

3) Define the standard translation of MLM Core models to a collection of two-level UML models.

4) Create a reference implementation of MLM Core.

5) Create model transformation specifications between MLM Core and the current MLM frameworks.

MLM Core would at first aim at unification. The next step could be a standardization effort. Such efforts are timeconsuming and could be detrimental to scientific progress. We argue that is is still worthwhile. Mapping existing MLM approaches to MLM Core opens the door to interoperability of models, and also to a uniform semantics of MLM models. The discussion triggered by the past MULTI challenges indicates that such mappings exist and are relatively easy to define.

There shall still be special constructs of existing or future MLM approaches that are not supported by the small set of constructs of MLM Core. If those constructs have a significant utility, then they should eventually be integrated into a new version of MLM Core.

\section{Strengthening the Community}

We believe that a strong community requires that its members know the work of others. Particular approaches are often, if at all, only known from a few publications. That may not be sufficient to develop a deeper appreciation.

Proposal: We should use community events such as the MULTI workshops or future Dagstuhl seminars to devote more time to present and discuss individual approaches and tools
In addition, we propose the following measures to strengthen the community and its recognition:

We regard developing and maintaining a unified representation of essential commonalities as a prerequisite for the further evolution of the field. However, the dissemination of MLM requires further measures.

To demonstrate the benefits of MLM, we need to develop illustrative examples and convincing use cases. To create awareness among outsiders, it should be useful to prepare narratives that focus on specific aspects of MLM and adapt them for various target groups, such as people with and without a background in modeling and software development.

In the long run, MLM can be established as a recognized and widely used modeling paradigm only, if we succeed in qualifying students accordingly. Therefore, we need to develop common recommendations for integrating MLM in existing curricula (lectures, tutorials). Since lecturers with the required expertise are rare, it might be useful to produce educational videos.

The design of multi-level models can be a demanding activity, which is suited to discourage many. To overcome this obstacle, MLM methods are needed. These are conceivable on different levels, like a general MLM method that guides the use of general-purpose modeling languages and more specific methods that relate to certain DSMLs developed within a multi-level languages architecture. To increase the visibility of MLM and to develop more elaborate evaluations of its benefits and potential drawbacks, there is need for larger research projects. Only larger projects that have the appropriate resources enable the development of models/systems that are clearly beyond "toy" examples. To that end, we need to submit research proposals to funding organizations like Deutsche Forschungsgemeinschaft or National Science Foundation.

We believe that, in addition to the above measures, it is essential for further promoting MLM to act as a strong community. Among other things that suggests to promote competition inside the community, and unity toward the outside. The latter would include that we jointly prepare research proposals and that we support the case of MLM within reviewing boards that lack a proper appreciation of it. Competition depends on critique. But for critique to work as a promoter of scientific progress, it should be appreciative and aimed at convincing justification.

\section{E. Common Research Agenda}

MLM offers numerous inspiring opportunities for research. Even though we believe that successful research depends on researchers' cognitive interest, we also think that a common research agenda is suited to foster progress of the field as such and to increase the value of individual contributions. Apart from that, various research topics are so challenging that they can hardly be mastered by single groups alone. This is the case, e.g., for extending or integrating MLM languages with multilevel programming languages, the development of large multilevel models or software systems such as, e.g., a prototypical ERP system. 


\section{F. Bundling of resources}

If the implementation of the above cornerstones of the roadmap is successful, it will be possible to bundle resources to jointly pursue common research goals that go clearly beyond the capabilities of single groups. In an ideal case, the community would establish a common repository of models that would be subject of collaborative research and could serve as showcases at the same time. Bundling of resources would also help with developing use cases and example models to supplement a common curriculum.

\section{CONCLUSIONS}

After 20 years of active research, the MLM community is at a crossroad. The first option is to regard MLM as an academic research area aiming to understand fundamental principles. The second option is to put an effort into some unification that delivers the definition of a common core for MLM, which then may be incorporated in commercial modeling tools, and possibly integrated development environments. The second option is not mutually exclusive to the first options since different research groups may continue to propose advanced MLM constructs beyond the common core. We argue that the second option provides the greater opportunities to make MLM become relevant for the mainstream of object-oriented modeling and programming. However, unification should not turn into an obstacle for the further development of MLM. Therefore, it would, at best, be restricted to a core the allows for widely monotonic extensions.

Arguably, the conceptual distance between existing MLM proposal is rather small, at least when limiting the constructs. While this is a promising position to start with, more research is needed to develop a unified foundation that would, e.g., enable the exchange of models between different MLM tools.

This position paper is a call to the MLM community for reflection on the opportunities. The proposals in this paper are not set in stone. The very constructs of the MLM Core have to be agreed by the community before any standardization work can start. We believe that the MLM community should make such an effort now, if it desires to become relevant to the larger modeling community, and ultimately contribute to modeling tools that are applied in education and in the real world. A solid MLM standard opens further possibilities such as influencing development environments. It may also have a significant impact on programming paradigms, i.e. multi-level programming languages.

\section{REFERENCES}

[1] C. Atkinson and T. Kühne, "The essence of multilevel metamodeling," in UML 2001, ser. LNCS, vol. 2185. Springer, 2001, pp. 19-33. [Online]. Available: http://dx.doi.org/10.1007/3-540-45441-1_3

[2] J. J. Odell, Advanced object-oriented analysis and design using UML. Cambridge University Press, 1998, ch. Power types, pp. 23-32.

[3] R. C. Goldstein and V. C. Storey, "Materialization," IEEE Transactions on Knowledge and Data Engineering, vol. 6, no. 5, pp. 835-842, 1994.
[4] A. Pirotte, E. Zimányi, D. Massart, and T. Yakusheva, "Materialization: A powerful and ubiquitous abstraction pattern," in VLDB'94, Proc. 20th International Conference on Very Large Data Bases, September 12-15, 1994, Santiago de Chile, Chile, J. B. Bocca, M. Jarke, and C. Zaniolo, Eds. Morgan Kaufmann, 1994, pp. 630-641. [Online]. Available: http://www.vldb.org/conf/1994/P630.PDF

[5] M. Jarke, R. Gallersdörfer, M. A. Jeusfeld, M. Staudt, and S. Eherer, "ConceptBase - a deductive object base for meta data management," J. Intell. Inf. Syst., vol. 4, no. 2, pp. 167-192, 1995. [Online]. Available: http://dx.doi.org/10.1007/BF00961873

[6] C. Atkinson and T. Kühne, "Reducing accidental complexity in domain models," Software \& Systems Modeling, vol. 7, no. 3, pp. 345-359, 2008.

[7] U. Frank, "Multilevel modeling - toward a new paradigm of conceptual modeling and information systems design," Business \& Information Systems Engineering, vol. 6, no. 6, pp. 319-337, 2014. [Online]. Available: https://doi.org/10.1007/s12599-014-0350-4

[8] B. W. Volz, Werkzeugunterstützung für methodenneutrale Metamodellierung, Bayreuth, 2011.

[9] J. de Lara and E. Guerra, "Deep meta-modelling with metadepth," in Objects, Models, Components, Patterns, 48th International Conference, TOOLS 2010, Málaga, Spain, June 28 - July 2, 2010. Proceedings, ser. Lecture Notes in Computer Science, J. Vitek, Ed., vol. 6141. Springer, 2010, pp. 1-20. [Online]. Available: https://doi.org/10.1007/ 978-3-642-13953-6_1

[10] F. Macías, A. Rutle, V. Stolz, R. Rodríguez-Echeverría, and U. Wolter, "An Approach to Flexible Multilevel Modelling," Enterprise Modelling and Information Systems Architectures, vol. 13, pp. 1-35, 2018.

[11] B. Neumayr, K. Grün, and M. Schrefl, "Multi-level domain modeling with m-objects and m-relationships," in Conceptual Modelling 2009, Sixth Asia-Pacific Conference on Conceptual Modelling (APCCM 2009), Wellington, New Zealand, January 20-23 2009, ser. CRPIT, M. Kirchberg and S. Link, Eds., vol. 96. Australian Computer Society, 2009, pp. 107-116. [Online]. Available: http://crpit.scem.westernsydney. edu.au/abstracts/CRPITV96Neumayr.html

[12] B. Neumayr, C. G. Schuetz, M. A. Jeusfeld, and M. Schrefl, "Dual Deep Modeling: Multi-Level Modeling with Dual Potencies and its Formalization in F-Logic," Software and Systems Modeling, vol. 17, no. 1 , pp. 233-268, 2018.

[13] C. Atkinson, R. Gerbig, and T. Kühne, "Comparing multi-level modeling approaches," in Proceedings of the MULTI Workshop colocated with MoDELS 2014, Valencia, Spain, September 28, 2014, ser. CEUR Workshop Proceedings, C. Atkinson, G. Grossmann, T. Kühne, and J. de Lara, Eds., vol. 1286. CEUR-WS.org, 2014, pp. 53-61. [Online]. Available: http://ceur-ws.org/Vol-1286/p6.pdf

[14] J. P. A. Almeida, U. Frank, and T. Kühne, "Multi-level modelling (Dagstuhl Seminar 17492)," Dagstuhl Reports, vol. 7, no. 12, pp. 18-49, 2017. [Online]. Available: https://doi.org/10.4230/DagRep.7.12.18

[15] U. Frank and D. Töpel, "Contingent level classes: Motivation, conceptualization, modeling guidelines, and implications for model management," in Proceedings of the 23rd ACM/IEEE International Conference on Model Driven Engineering Languages and Systems: Companion Proceedings, E. Guerra and L. Iovino, Eds. New York, NY, USA: ACM, 2020, pp. 622-631.

[16] M. A. Jeusfeld, "DeepTelos for ConceptBase: A contribution to the MULTI process challenge," in 22nd ACM/IEEE International Conference on Model Driven Engineering Languages and Systems Companion, MODELS Companion 2019, Munich, Germany, September 15-20, 2019. IEEE, 2019, pp. 66-77. [Online]. Available: https: //doi.org/10.1109/MODELS-C.2019.00016

[17] T. Kühne, "A story of levels," in Proceedings of MULTI 2018, Copenhagen, 2018.

[18] T. Clark, C. Gonzalez-Perez, and B. Henderson-Sellers, "A foundation for multi-level modelling," in Proceedings of the MULTI Workshop co-located with MoDELS 2014, Valencia, Spain, September 28, 2014, ser. CEUR Workshop Proceedings, C. Atkinson, G. Grossmann, T. Kühne, and J. de Lara, Eds., vol. 1286. CEUR-WS.org, 2014, pp. 43-52. [Online]. Available: http://ceur-ws.org/Vol-1286/p5.pdf 